\title{
Usages et fonctions du matériel didactique : pratiques déclarées d'enseignants et de futurs enseignants d'histoire au secondaire
}

\author{
Benefits and challenges of active learning \\ classrooms in cegeps, according to teachers and students: \\ First-iteration results of a design-based study
}

Vincent Boutonnet Université du Québec en Outaouais

\section{ésumé}

Cette recherche vise à décrire et à analyser la façon dont de

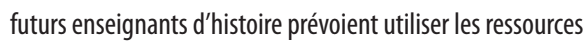
didactiques et les fonctions qu'ils attribuent à ces ressources pour exercer la pensée historienne comparativement à

des enseignants en exercice. Les futurs enseignants et les enseignants semblent partager plusieurs fonctions attribuées aux ressources didactiques, dont celles de référence et d'illustration. De plus, les dimensions essentielles de la pensée historienne semblent être rejetées (débat historiographique, analyse de sources contradictoires, etc.). Nous discutons de la réussite de la formation initiale de futurs enseignants qui devrait les conduire à choisir des fonctions plus critiques lors de l'enseignement de l'histoire au secondaire.

Mots-clés

Enseignement, histoire, secondaire, matériel didactique, futurs enseignants, pratiques enseignantes.

\section{Abstract}

This research aims to describe and analyze how history preservice teachers plan to use teaching resources and what functions they assign to these resources in order to foster historical thinking as compared to in-service teachers.

Preservice teachers and teachers seem to share several functions attributed to teaching resources, including reference and illustration. Moreover, the essential dimensions of historical thinking seem to be rejected (historiographic debate, analysis of contradictory sources, etc.). We discuss the success

of preservice teachers training who should choose more critical functions assigned to history teaching in high school.

Keywords

History teaching, high school, teaching resources, preservice teacher, teaching practices.

\section{Problématique}

L'enseignement ${ }^{1}$ de l'histoire au secondaire fait régulièrement couler de l'encre sur la place publique, en particulier lorsqu'il est question de l'histoire nationale. En effet, il est toujours question du choix des contenus historiques, du type d'enseignement, de l'intégration de groupes minorisés (c'est-à-dire les immigrants, les autochtones, les femmes, etc.) ou du recours à une trame narrative nationaliste (Éthier, Boutonnet, Demers et Lefrançois, 2017). À ces questions importantes s'ajoute aussi la qualité de la formation initiale des futurs enseignants d'histoire. Certains réclament régulièrement plus de cours d'histoire durant le cursus universitaire afin de pallier la faible maîtrise des contenus historiques par les futurs enseignants (Bédard et D'Arcy, 2011; Lavallée, 2012).

Il importe assurément de s'intéresser à la formation initiale puisque celle-ci contribue à la formalisation des pratiques d'enseignement qui pourraient devenir lacunaires, particulièrement dans les premières années d'exercice (Martineau, Gervais, Portelance et Mukamurera, 2008). En outre, les pratiques d'enseignement en histoire au secondaire restent magistrocentrées et traditionnellement fondées sur la transmission de connaissances historiques dites essentielles (Boutonnet, 2015a; Demers, 2011) alors que le programme d'histoire au secondaire vise à exercer des habiletés de critique, de comparaison et de débat en classe autant dans l'ancien programme (Ministère de l'Éducation, du Loisir et du Sport, 2007) que le nouveau ministère de l'Éducation et de l'Enseignement supérieur (MEES, 2017)2 Nous précisons toutefois que cette recherche a été menée lorsque le programme de 2006 était en vigueur, nous y reviendrons dans le cadre méthodologique. 
Dès lors, la formation initiale des futurs enseignants d'histoire au secondaire semble un enjeu important du système éducatif québécois, en particulier si on souhaite des pratiques innovantes et variées. Or, la formation initiale des futurs enseignants d'histoire ne semble pas toujours modifier leurs représentations quant à l'enseignement de l'histoire (Monte-Sano, 2011; Reitano et Green, 2013). Il ne faut probablement pas en être surpris si, comme étudiants à l'université, ils expérimentent le plus souvent des cours magistraux qui requièrent peu de recherches et d'analyses de sources historiques (Lévesque, 2016). Ainsi, décrire les représentations des futurs enseignants québécois d'histoire au secondaire est nécessaire afin de comprendre leur évolution et leur incidence éventuelle dans la formalisation de leurs pratiques futures. Plus précisément, nous nous intéressons aux fonctions et aux usages des ressources didactiques qui pourraient favoriser le recours à des documents variés et leur critique en classe. Pour ce faire, nous utiliserons aussi des données issues de notre recherche doctorale qui portait sur des objectifs similaires, mais avec comme échantillon des enseignants d'histoire au secondaire.

Nous proposons donc de comparer et d'analyser : 1) l'usage des ensembles didactiques d'histoire et des ressources complémentaires par les enseignants et les futurs enseignants au secondaire; 2) les fonctions attribuées aux ressources par les enseignants et les futurs enseignants au secondaire.

\section{Cadre théorique}

Si la formation initiale semble un facteur déterminant dans la formalisation des pratiques d'enseignement, nous devons aussi souligner que le processus d'appropriation du savoir historique au moyen de documents variés peut différer entre l'utilisation effective des enseignants et les intentions de transmission des auteurs (Apple, 1992; Wertsch, 1997). Au sein de cette tension, il faut alors considérer quels documents sont utilisés, selon quelles modalités et pour quelles finalités. Nous proposons d'examiner cette triple médiation à l'aune de trois concepts essentiels à la poursuite de nos objectifs : l'exercice de la méthode historique, l'intervention éducative et l'usage des ressources didactiques en classe d'histoire au secondaire.

\section{L'exercice de la méthode historique}

Le développement d'habiletés spécifiques à l'histoire n'est pas inné et ne progresse pas au même rythme pour tous les élèves (Lee et Shemilt, 2003; Wineburg, Martin et Monte-Sano, 2012). Il importe alors de concevoir des situations d'apprentissage qui soient dédiées à cette finalité et qui soient adaptées aux besoins des élèves. Cet ensemble d'habiletés fait référence à la pensée historienne, c'est-à-dire aux modes de pensée et aux méthodes de travail spécifiques à la discipline historique (Prost, 1996). Cela dit, la liste d'habiletés à maîtriser varie selon les auteurs (Martineau, 1999; Seixas et Morton, 2013; Wineburg, 2001), mais tous s'entendent sur la nécessité de recourir à des sources historiques variées et parfois contradictoires afin d'offrir aux élèves la possibilité de s'exercer à la critique de documents et à l'argumentation historique. C'est l'euristique de la source (Demers, Lefrançois et Éthier, 2010; Wineburg, 2001) qui peut se décliner en quatre étapes : attribuer le caractère d'une source historique à une trace du passé (capacité à identifier la signifiance de la source), la lecture attentive par la critique de la source (capacité à inférer et à critiquer le contenu, mais aussi les intentions de l'auteur), la contextualisation (mobiliser des connaissances sur le contexte historique) et la corroboration 
(comparer plusieurs points de vue par l'entremise de plusieurs sources). L'euristique de la source est la mise en œuvre pratique de la méthode historique qui conçoit un rapport au savoir dans une perspective constructiviste et interprétative des savoirs historiques. Il ne s'agit donc pas de simplement apprendre et mémoriser des dates ou des faits importants. En fait, les élèves du secondaire sont non seulement capables de critiquer des textes historiques, mais ils sont aussi capables de débattre, de s'interroger et d'argumenter de manière rigoureuse lorsque les activités qui leur sont proposées sont consacrées à exercer la méthode historique, lorsqu'ils ont recours à une variété de documents et lorsque cet exercice est soutenu par l'enseignant (Nokes, 2011; Reisman, 2012; van Boxtel et van Drie, 2017). En outre, cet exercice sera consolidé si le savoir historique est problématisé et si les élèves sont engagés dans une démarche d'enquête en lien avec la méthode historique (Yelle et Déry, 2017).

\section{L'intervention éducative}

Le succès de la progression de la pensée historienne chez les élèves dépend alors des activités expérimentées en classe, des documents mobilisés et par conséquent de la planification de l'enseignant. Afin de comprendre les pratiques d'enseignement, plusieurs ont suggéré des modèles théoriques de classification ou des typologies pour distinguer des activités de médiation du savoir en classe. Un modèle qui nous apparaît fécond est celui de l'intervention éducative théorisé par Not (1979) et repris plus au Québec par Lenoir (1991) pour l'enseignement des sciences humaines. L'intérêt de ce modèle réside dans la prise en compte de la diversité des pratiques enseignantes et la relation qu'ils mettent en place entre l'élève et les différents outils mis à leur disposition, dont les ressources utilisées en classe (par exemple, une source historique). Ce modèle conçoit quatre modulations de l'apprentissage: l'hétérostructuration traditionnelle (MIE1), l'hétérostructuration de type coactif (MIE2), l'autostructuration (MIE3) et l'interstructuration cognitive (MIE4). Le MIE1 conçoit le savoir comme préexistant et extérieur aux connaissances de l'élève. Le rôle de l'enseignant est de révéler ce savoir le plus souvent par un exposé magistral. L'élève est la plupart du temps passif puisqu'on attend seulement qu'il écoute attentivement et pose des questions lorsque nécessaire. C'est la pédagogie transmissive. Le MIE2 attribue davantage la construction du savoir à l'élève, même si l'enseignant reste celui qui structure les activités et le chemin à suivre pour progresser. C'est la pédagogie de la découverte où l'enseignant fait découvrir le savoir au fur et à mesure d'un cheminement prescrit. L'usage de cahiers d'exercices est un exemple évocateur de cette pratique d'enseignement. Le MIE3 favorise la pédagogie naturelle ou non directive. Le rôle de l'enseignant est de laisser l'élève expérimenter, se questionner ou tâtonner jusqu'à construire un savoir qui sera par la suite validé. En dernier lieu, le MIE4 tente de pallier les lacunes du MIE2 et MIE3 : l'élève participe de manière plus autonome à la structuration du savoir et peut emprunter différents chemins pour y arriver. Le rôle de l'enseignant est de guider et de soutenir la recherche alors que l'élève doit se questionner et structurer ses propres connaissances; c'est la pédagogie interactive de la recherche.

L'enseignement de l'histoire au Québec, comme dans de nombreux pays, reste encore très magistrocentré par l'entremise de pratiques transmissives ou encore structurantes comme la pédagogie de la découverte (Boutonnet, 2015b; Lautier et Allieu-Mary, 2008; Nokes, 2010). Lélève est généralement passif, en position d'écoute, de prise de notes et l'enseignant structure et transmet le savoir essentiel à apprendre, voire à mémoriser. De plus, ces pratiques d'enseignement sont influencées par les représentations 
des enseignants sur l'apprentissage de l'histoire et leurs positions épistémiques qui laissent peu de la place à la critique, à l'argumentation ou au questionnement (Bouhon, 2012; Demers, 2011; Moisan, 2010). Selon les enseignants, le savoir devrait être graduellement acquis par les élèves sur la base d'un corpus de dates et de faits historiques importants à connaître avant d'exercer des habiletés propres à la pensée historienne (Boutonnet, 2015b; Demers, 2011). Les pratiques enseignantes, ou autrement dit l'intervention éducative, sont essentielles à considérer dans l'exercice de la méthode historique par les élèves. Dans ces conditions, le rôle de la formation initiale et continue semble important pour le renouvellement et la transformation des pratiques d'enseignement afin d'assurer des pratiques intégrant authentiquement la démarche d'enquête par l'exercice de la méthode historique.

\section{L'usage des ressources didactiques}

Cependant, peu de recherches se sont intéressées à l'usage des ressources didactiques pour favoriser l'exercice de la méthode historique alors que leurs propositions didactiques pourraient influencer les pratiques d'enseignement autant au primaire (Araujo-Oliveira, 2012; Lebrun, 2001) qu'au secondaire (Niclot et Aroq, 2006; Repoussi et Tutiaux-Guillon, 2012; Vargas, 2006). En outre, trois tendances sont identifiables sur l'usage des ressources en classe : 1) la place importante du manuel dans le dispositif d'intervention éducative des enseignants (Blaser, 2007; Haydn, 2011; Vinterek, 2010); 2) un usage morcelé et diversifié le plus souvent comme illustration du récit de l'enseignant ou comme banque d'exercices (Baquès, 2005; Braxmeyer, 2007; Nokes, 2010); 3) un usage relatif aux représentations des enseignants et à une structuration du savoir contrôlée par leur récit (Helgason, 2010; Jadoulle, 2015; Kon, 1995; Zahorik, 1991). C'est pourquoi nous postulons que l'exercice de la méthode historique et de ses habiletés spécifiques dépendra des ressources utilisées, mais surtout de l'intervention éducative des enseignants.

Ce cadre théorique nous conduit à formuler trois types d'usages des ressources en lien avec les représentations des enseignants et leurs modalités d'intervention éducative : 1) intensif; 2) extensif; 3) critique. Cette typologie est fondée sur une étude multicas et validée par la comparaison de pratiques déclarées et constatées d'enseignants d'histoire au secondaire (Boutonnet, 2015a, 2015b). Le type intensif s'appuie presque intégralement sur les propositions didactiques et le contenu suggérés par les ressources didactiques. L'enseignant en dévie rarement et par conséquent le manuel ou le cahier d'exercices détermine fortement la planification et la progression des apprentissages. Le type extensif se caractérise par une autonomie plus grande de l'enseignant vis-à-vis des propositions des ressources didactiques. Les ressources seront utilisées régulièrement, mais de manière sélective et la plupart du temps une variété de documents supplémentaires seront affichés afin d'illustrer le récit de l'enseignant. Le type critique incarne l'exercice de la pensée historienne, en proposant non seulement une variété de documents, mais en favorisant surtout les habiletés liées aux euristiques et à l'argumentation historique. Ces trois types nous permettent de concevoir des pratiques d'enseignement variées selon les représentations des enseignants ou des futurs enseignants quant aux fondements de la discipline historique et à ce qu'il est possible de réaliser en classe. 


\section{Méthodologie}

La présente étude est une recherche exploratoire qui reprend le cadre méthodologique de la thèse doctorale axée uniquement sur les pratiques d'enseignants d'histoire au secondaire (Boutonnet, 2015a, 2015b). Cet article considère donc deux cas qui seront comparés selon deux populations distinctes (enseignants et futurs enseignants d'histoire au secondaire) qui ont suivi un devis de recherche semblable : 1) un questionnaire sur l'usage des ressources et leurs fonctions attribuées par les répondants; 2) la réalisation d'un schéma conceptuel représentant l'usage des ressources didactiques afin de favoriser l'enseignement de l'histoire au secondaire; 3) un entretien de suivi et d'explicitation mené après la passation du questionnaire et la réalisation du schéma conceptuel. Les résultats pour l'échantillon des enseignants sont issus de la thèse doctorale (Boutonnet, 2015a, 2015b) alors que les résultats pour l'échantillon des futurs enseignants ont été collectés durant l'automne 2015. C'est donc la raison pour laquelle cette recherche ne s'applique qu'au contexte du programme d'histoire et d'éducation à la citoyenneté antérieur en vigueur de 2006 à 2017. Afin d'être plus exhaustif dans notre analyse, l'objet de cet article est de comparer uniquement les résultats du questionnaire de futurs enseignants $(n=58)$ aux questions identiques adressées aux enseignants $(n=81)$ dans le cadre de la recherche doctorale.

Le questionnaire se compose de deux parties : la première comporte six questions sur les fonctions attribuées aux ressources par les répondants qui doivent choisir pour chaque question deux énoncés se rapprochant de leurs représentations et en rejeter deux autres qui sont les plus éloignés; la deuxième comporte cinq questions sur les usages déclarés de ces ressources selon une échelle de type Likert à cinq points (de jamais à très souvent). La première partie est utile pour mieux circonscrire les représentations puisque les répondants ne choisissent pas uniquement des énoncés qui leur conviennent, mais doivent aussi en rejeter (Vergès, 2001). En outre, nous avons formulé les énoncés au regard des types d'usages identifiés dans le cadre théorique et le questionnaire a été validé lors de la recherche doctorale par un codage inversé et une évaluation par un comité d'experts. Ainsi, il y a deux énoncés pour chaque type, donnant six énoncés pour chaque question de la première partie. Ce type de questions constitue des résultats sous la forme d'un indice où 1 représente un choix absolu d'un énoncé et où -1 représente un rejet absolu d'un énoncé. La deuxième partie est plus classique et vise à décrire simplement l'importance de certaines ressources au regard des pratiques d'enseignement déclarées.

Les limites de la recherche sont liées à l'échantillon limité et de convenance qui contraignent la généralisation des résultats. En outre, les données ne sont que déclarées et nous soulignons l'écart qui se constate parfois avec des pratiques réelles ou constatées en situation de classe (Boutonnet, 2015b; Bouhon, 2012). Nous rappelons aussi que cet article ne se concentre que sur les données en lien avec le sondage qui peut effectivement mener à différentes interprétations par les participants selon les énoncés choisis. Cela dit, c'est une limite inhérente aux questionnaires quantitatifs même si le questionnaire est validé. Malgré ces limites, les deux populations présentent des profils variés (enseignants novices et expérimentés provenant du système public ou privé alors que les futurs enseignants proviennent des universités francophones du Québec et sont inscrits de la $1^{\text {re }}$ à la $4^{\mathrm{e}}$ année du BES) et suffisamment riches pour assurer la validité des résultats et de la discussion. 


\section{Résultats}

\section{Les types d'usages des ressources didactiques}

L'ensemble des réponses aux questions de la première partie du questionnaire permet de constituer un score individuel selon les choix et les rejets d'énoncés. Les énoncés sont pondérés selon les types avec les énoncés du type critique qui obtiennent deux points par choix, un point par choix pour les énoncés du type extensif alors que le type intensif n'obtient aucun point par choix. Lorsqu'il y a rejet des énoncés, le pointage est négatif, par exemple - 1 point pour les énoncés de type intensif. Par conséquent, le score individuel peut atteindre un maximum de 4 points ou un minimum de -4 points.

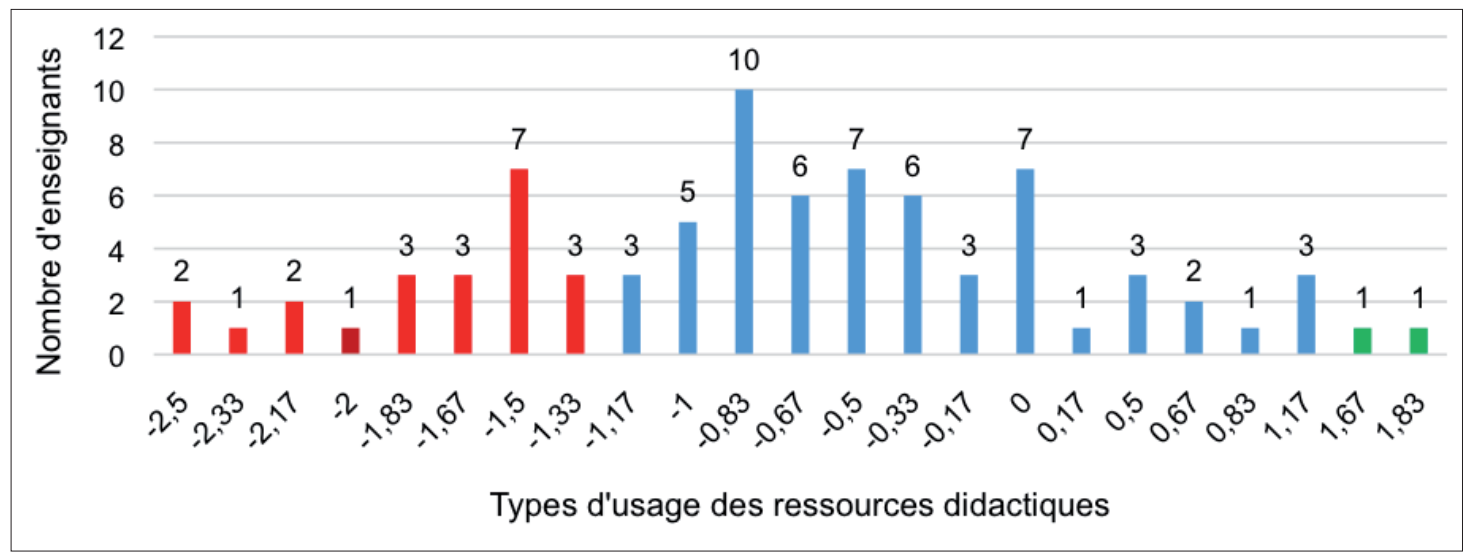

Figure 1

Répartition des enseignants $(n=81)$ selon leur type.

Cette première figure illustre la répartition des enseignants selon leur type d'usages des ressources didactiques. On peut observer une très faible répartition du type critique (en vert, $n=2$ ) alors que le type extensif est le plus représenté (en bleu, $n=57$ ), suivi par le type intensif (en rouge, $n=22$ ). Ces résultats correspondent aux constats identifiés dans le cadre théorique, à savoir que les enseignants ont un usage sélectif des ressources didactiques selon leurs besoins et généralement afin d'illustrer leur propos.

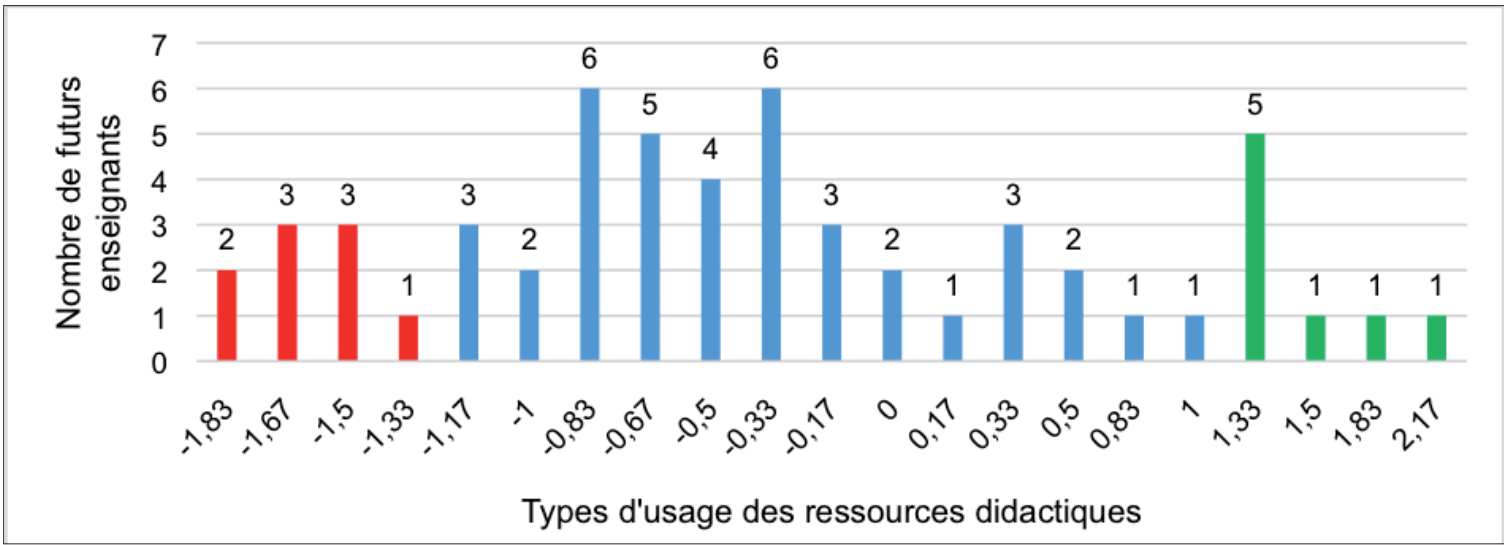

Figure 2

Répartition des futurs enseignants $(n=58)$ selon leur type 
Les futurs enseignants présentent une répartition très différente : il y a un nombre plus élevé du type critique (en vert, $n=8$ ), un nombre moins élevé du type intensif (en rouge, $n=9$ ) et une répartition très importante pour le type extensif (en bleu, $n=41$ ). Ce résultat semble illustrer que les futurs enseignants expriment des représentations plus proches de l'exercice de la pensée historienne. Cela signifie-t-il que la formation initiale favorise l'apparition du type critique? Ces données semblent encourageantes, mais les données sont, d'une part, fortement influencées par les réponses à la question 5 qui présente un indice positif très élevé et, d'autre part, les données présentent un pic exceptionnel de cinq répondants avec le score de 1,33 alors qu'on observe bien une concentration du type extensif pour les scores de $-0,83$ à $-0,33$ (concentration d'ailleurs assez semblable pour les enseignants - voir figure 1). Nous verrons que lorsque nous considérons les résultats par énoncés, les représentations sont somme toute proches de celles des enseignants.

\section{Les fonctions attribuées aux ressources didactiques}

En effet, les futurs enseignants ont tendance à choisir de manière légèrement supérieure $(0,59)$ l'énoncé «le rôle du manuel est de montrer des ressources iconographiques ou textuelles » comparativement aux enseignants $(0,56)$. Cependant, les enseignants choisissent largement l'énoncé de type intensif « d'exposer les faits et les personnages importants » $(0,47)$ alors que les futurs enseignants le choisissent moins $(0,22)$. En outre, le rejet de l'énoncé de type critique "le rôle du manuel est d'exercer aux fondements de la discipline historique » est assez semblable pour les futurs enseignants $(-0,50)$ et les enseignants $(-0,43)$. Cela dit, le deuxième énoncé rejeté est différent selon la population : les enseignants $(-0,48)$ rejettent « le rôle du manuel est de proposer des activités et des démarches d'enseignement » alors que les futurs enseignants $(-0,29)$ rejettent « le rôle du manuel est d'encourager l'autonomie des élèves ». Cette première question semble affirmer la fonction de référence et d'illustration du manuel tout en écartant la fonction d'exercer aux fondements de la discipline historique pour les deux populations. Cela continue d'assoir des représentations liées à des pratiques transmissives et peu critiques apparentées au type d'usage intensif.

Sur le récit du manuel, les enseignants préfèrent la fonction de "présenter une chronologie des évènements majeurs » $(0,47)$ avant de "proposer des sources primaires conflictuelles » $(-0,35)$ alors que le travail sur les sources historiques est essentiel pour développer la pensée historienne. Pour les futurs enseignants, le manuel devrait aussi servir à "présenter une chronologie des évènements majeurs » $(0,48)$ alors qu'ils rejettent largement la fonction " d'exposer des débats historiographiques » $(-0,43)$. Encore une fois, les choix ou rejets sont très semblables pour les deux populations et sont aussi très cohérents avec la question précédente : les activités d'analyse ou de critique sont secondaires, ce qui importe est d'apprendre un récit historique chronologique.

Compte tenu des résultats à la première question qui semblent contester le rôle du manuel pour exercer aux fondements disciplinaires, il n'est pas surprenant de constater l'ambivalence des deux populations représentée par des indices proches de 0 pour l'ensemble des énoncés à la question sur les fonctions attribuées au manuel pour exercer à la pensée historienne. En fait, un seul énoncé se démarque pour les enseignants qui pensent que le manuel permet « d'expliquer les causes et les conséquences des évènements " $(0,57)$ et moindrement pour les futurs enseignants $(0,28)$. Les représentations des enseignants sont stables au regard d'une vision de la discipline historique fondée sur un récit unique et 
chronologique que l'on peut rarement remettre en cause. En outre, les futurs enseignants ne présentent que des indices proches de 0 , ce qui pourrait être symptomatique d'une formation initiale et pratique qui n'est pas encore complétée. Cela pourrait influencer des représentations encore parfois peu formalisées pour certaines dimensions.

À la question «Lorsque vous nêtes pas satisfaits du traitement de certains sujets par votre manuel » les deux populations s'accordent pour dire quelles préfèrent «utiliser d'autres sources à la place ou en complément du manuel» ( 0,75 pour les enseignants et 0,79 pour les futurs enseignants) et « sélectionner seulement les parties les plus pertinentes » $(0,63$ et 0,59). Les enseignants et les futurs enseignants s'entendent pour rejeter l'activité de « critiquer ouvertement le manuel» $(-0,44$ et $-0,53)$. Cependant à l'activité « suivre le manuel tout de même », les enseignants $(-0,44)$ le rejettent beaucoup moins que les futurs enseignants $(-0,90)$. Ces résultats confirment la tendance des deux populations à avoir un usage clairement sélectif et éclaté du manuel qui s'apparente au type extensif. Cela dit, le rejet massif de « suivre le manuel » manifeste une certaine capacité des futurs enseignants à écarter certaines ressources didactiques lorsque la qualité leur semble problématique.

Les futurs enseignants identifient un avantage important à l'usage des ressources, celui « d'être un bon outil de référence » $(0,66)$ alors qu'ils considèrent peu la qualité « de prendre en compte les besoins particuliers des élèves » $(-0,34)$. Les enseignants identifient aussi la qualité « d'être un bon outil de référence » $(0,63)$ alors que la qualité qu'il leur semble moins essentielle est celle de "guider l'élève dans l'exercice de techniques » $(-0,49)$ spécifiques à l'usage de documents variés.

Les limites identifiées par les répondants obtiennent des indices relativement peu élevés. En effet, la principale limite est " de présenter un récit peu ou pas assez exhaustif » $(0,32)$ ainsi que « de contenir peu ou pas assez de sources premières » $(0,33)$ pour les futurs enseignants. Ce résultat est un peu surprenant particulièrement parce que les futurs enseignants ont aussi rejeté à la deuxième question la fonction du manuel de "présenter des sources primaires dont le contenu est contradictoire " (0,34). Peut-être que la dimension de "présenter des sources contradictoires » est moins essentielle pour les futurs enseignants, même si utiliser des sources historiques reste assez important. Cela dit, on peut se questionner sur l'efficacité des activités si les élèves sont rarement confrontés à des sources contradictoires. Nous pourrions y voir encore la prééminence d'apprendre un récit uniforme qui est peut-être plus facile à mémoriser pour les élèves, mais qui exerce moins les habiletés à critiquer et à argumenter. Les enseignants présentent des représentations très semblables en identifiant la limite "de présenter un récit peu ou pas assez exhaustif » $(0,25)$ alors que ce qui semble moins important à considérer est la limite « de guider peu ou pas assez l'élève dans des techniques » $(-0,38)$. 


\section{Les usages des ressources didactiques}

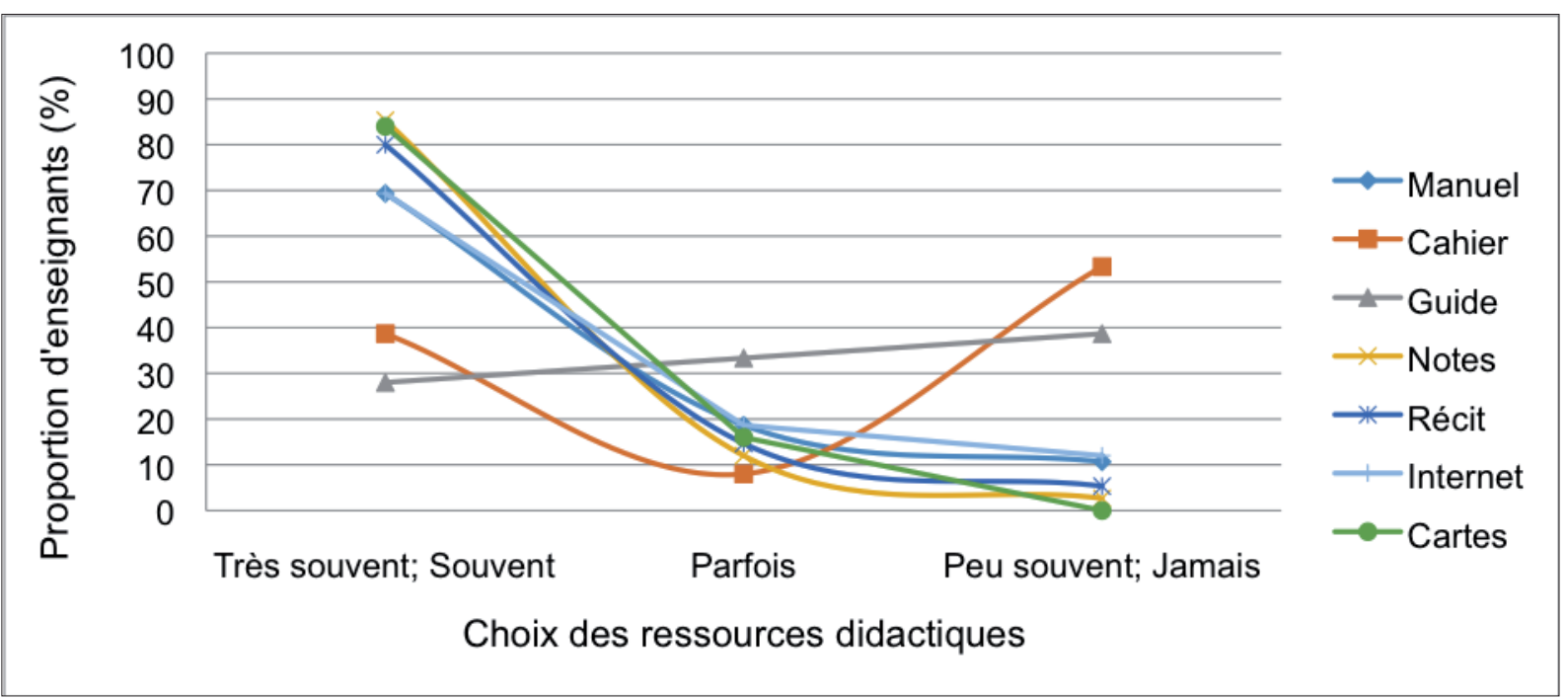

Figure 3

Choix des ressources didactiques par les enseignants.

La figure 3 illustre les ressources choisies par les enseignants pour en faire un usage didactique à la deuxième partie du questionnaire. Plusieurs données sont intéressantes. Tout d'abord, les enseignants semblent peu considérer le guide de l'enseignant qui accompagne les manuels. Ce n'est pas insolite puisque les enseignants auraient un usage sélectif du manuel selon leurs besoins, ce qui signifie de s'écarter régulièrement des propositions didactiques du manuel et de son guide. Cela dit, ils déclarent aussi utiliser très souvent ou souvent le manuel ou les notes de cours en classe. En outre, on remarque aussi la présence importante du récit en classe, c'est-à-dire de l'exposé magistral, dans les pratiques déclarées des enseignants. Autre fait intéressant, l'usage du cahier d'exercices présente une cloche inversée signifiant que certains enseignants ont tendance à le privilégier alors que d'autres l'écartent complètement. C'est une position plutôt ambivalente, soit ils préfèrent le cahier, soit ils le rejettent. En dernier lieu, les cartes et internet occupent une place importante dans le choix des ressources par les enseignants. 


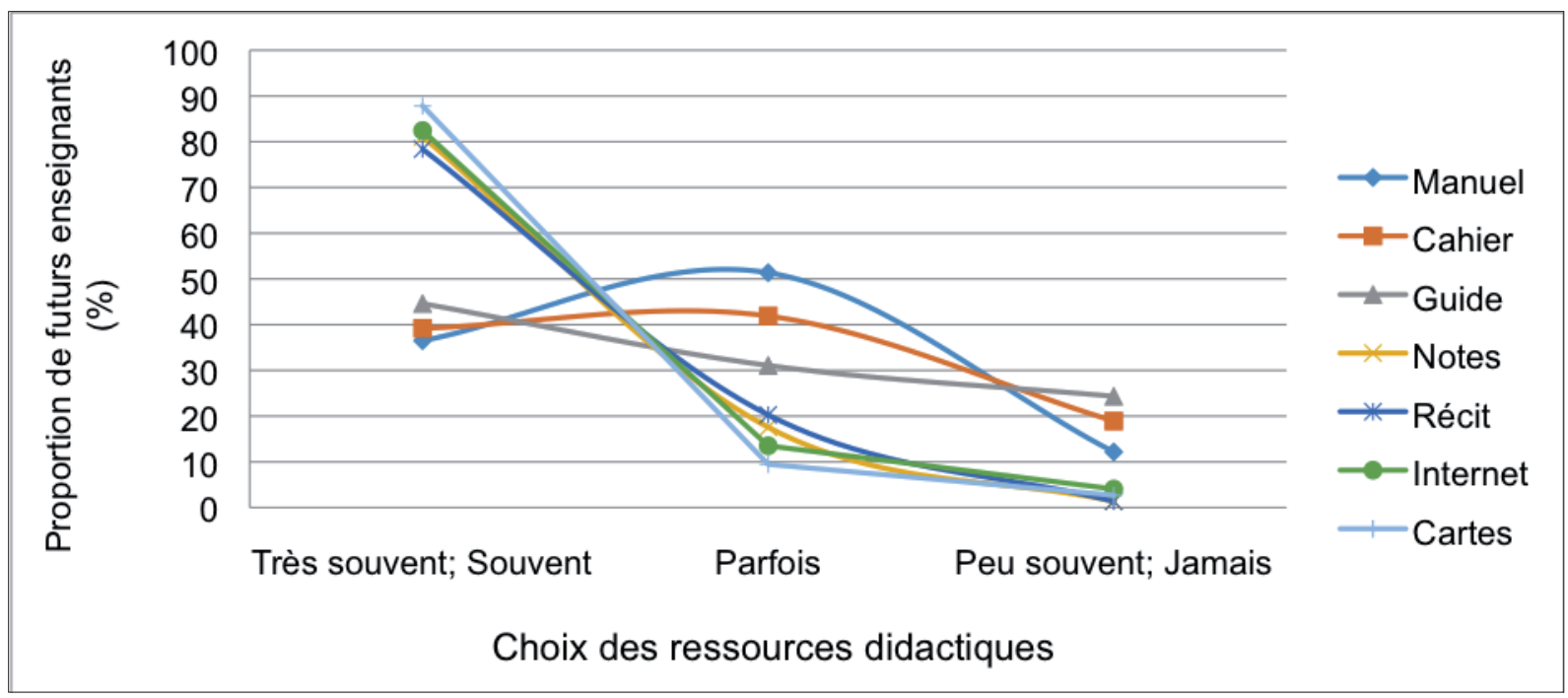

Figure 4

Choix des ressources didactiques par les futurs enseignants.

Les futurs enseignants présentent un profil assez différent de celui des enseignants. Tout d'abord, on souligne la même importance attribuée aux notes de cours, au récit magistral, aux cartes et à internet. Cependant, l'usage d'internet est relativement plus important pour les futurs enseignants ( $82 \%$ très souvent et souvent) que pour les enseignants (69\% très souvent ou souvent). Cet écart de presque 10 points est peut-être une question de génération, mais nous pensons que ce résultat est à relativiser. Ensuite, trois données sont aussi différentes pour les futurs enseignants. Premièrement, l'usage du manuel semble moins important avec une courbe en cloche qui marque un usage moins extensif que les enseignants. Deuxièmement, le guide de l'enseignant qui accompagne le manuel semble plus important pour les futurs enseignants avec tout de même un usage important pour $45 \%$ (très souvent et souvent) des futurs enseignants contre $28 \%$ (très souvent et souvent) des enseignants. Finalement, l'usage du cahier d'exercices semble aussi plus important pour les futurs enseignants avec une courbe légèrement en cloche. Ces deux derniers constats pourraient signifier la prégnance des propositions didactiques du cahier d'exercices ou de guide de l'enseignant afin de planifier leurs activités alors que les enseignants tendent à les délaisser. Ce constat correspond avec ceux de Vargas (2006) qui souligne que souvent le matériel didactique fait office d'un plus grand soutien pour les enseignants novices qui tendent à le délaisser au fur et à mesure de leurs expériences. 


\section{Conclusion}

Les pratiques d'enseignement relatives à l'usage des ressources didactiques sont semblables pour les enseignants et les futurs enseignants. En effet, les ressources didactiques servent principalement de référence et d'illustration pour l'exposition de faits, de grands personnages et d'une chronologie des évènements. La méthode historique ne semble pas une priorité puisque ses dimensions les plus essentielles sont rejetées par les deux populations (exercice aux fondements de la discipline historique, usages de sources contradictoires ou de débats historiographiques). On constate alors une cohérence et une stabilité des représentations pour les deux groupes. De prime abord, la formation initiale ne semble avoir que peu d'influence sur les représentations des futurs enseignants. Comment alors expliquer des similitudes entre des enseignants expérimentés et de futurs enseignants en formation?

Les pressions sociales et institutionnelles permettraient d'expliquer en partie la persistance de représentations traditionnelles alors que les ressources et les moyens d'enseignement se diversifient sans favoriser l'exercice authentique de la méthode historique. La pratique, ou l'habitus selon Bourdieu (1994), se reproduit au sein d'un groupe par des mécanismes d'autorégulation. C'est par ce processus que les nouveaux membres d'un groupe seront accueillis et socialisés selon les pratiques communes et acceptées dans une institution (par exemple, une école ou une université). Nous observons donc que la formation initiale ainsi que l'insertion professionnelle de futurs enseignants restent des défis importants.

D'ailleurs, plusieurs chercheurs observent des obstacles et des résistances à la formation pratique en contexte de stage dans le milieu scolaire (Altet, Desjardins, Étienne, Paquay et Perrenoud, 2013). Cette problématique pourrait s'expliquer par la tension, voire l'opposition, entre les savoirs pratiques $\mathrm{du}$ milieu professionnel et les savoirs théoriques du milieu universitaire. Les stagiaires semblent reconnaître l'utilité de leur formation initiale, mais expriment en même temps la primauté du savoir pratique développé par les enseignants en exercice ainsi que le faible recours aux savoirs théoriques (Gervais et Leroux, 2011; Guibert, 2013). En fait, certains stagiaires naviguent par des ruptures et des recompositions de leurs pratiques d'enseignement au cours de leurs stages afin de s'ajuster à ces tensions entre pratique et théorie (Malo, 2011). Il semblerait même que les futurs enseignants en stage se réfèrent au manuel ou à l'enseignant associé pour faire approuver leurs pratiques (Therriault et Harvey, 2011).

Pourtant, cette opposition entre formation pratique et théorique semble insuffisante pour expliquer la réussite mitigée de la formation initiale de futurs enseignants (Chaubet et Gervais, 2014; Korthagen, Loughran et Russell, 2006). L'adéquation entre les milieux de formation et les différents intervenants lors de la formation initiale et pratique serait une piste de solution. En effet, la formation initiale nest pas uniforme dans nos institutions universitaires. S’il existe des compétences professionnelles prescrites pour l'ensemble des universités québécoises, il n'existe pas de formation-cadre pour les atteindre, les évaluer et encore moins pour les développer au niveau disciplinaire (par exemple, que signifie « être un professionnel héritier, critique et interprète d'objets de savoirs ou de culture » spécifiquement pour les futurs enseignants d'histoire?). 
Les résultats de cette étude nous sensibilisent au fait que la formation initiale ne déconstruit pas assez des représentations traditionnelles issues du milieu scolaire ou social. L'habitus serait peut-être une dimension intéressante à examiner tout en analysant les conceptions initiales des futurs enseignants afin de mieux comprendre leur progression pendant leur formation pratique et théorique afin d'identifier précisément les lieux de résistance et d'adapter nos formations universitaires en conséquence. Les changements curriculaires sont souvent des moments de débats et de résistances vifs dans la société sans toutefois garantir une transformation en profondeur des pratiques d'enseignement autant au niveau secondaire qu'au niveau postsecondaire (Lenoir, 2011; Morton, 2011). La formation initiale mais aussi la formation continue devraient rester des priorités importantes pour le gouvernement et nos institutions universitaires si nous espérons nous donner les moyens de former du personnel enseignant critique, autonome et innovateur.

\section{Notes}

1 Cette recherche a été subventionnée par le Fonds institutionnel de développement de la recherche et de la création de l'Université du Québec en Outaouais, programme de soutien aux nouveaux professeurs de l'UQO.

2 Lors de la recherche présentée par cet article, le programme d'histoire nationale de 2006 était alors en vigueur, nous utiliserons donc les visées et les compétences prescrites par ce programme. Depuis, le nouveau programme d'histoire du Québec et du Canada pour la $3^{\mathrm{e}}$ et la $4^{\mathrm{e}}$ année du secondaire a été approuvé par le MEES lors de la rentrée 2017. Il en découle plusieurs changements substantiels : un étalement chronologique des savoirs sur les deux années (alors qu'auparavant la $3^{\mathrm{e}}$ année était chronologique et la $4^{\mathrm{e}}$ année thématique), un redécoupage des réalités sociales à l'étude, l'intégration de la précision des connaissances dans le programme (auparavant la progression des apprentissages était présentée dans un document à part) et le remodelage des compétences disciplinaires à travailler avec les élèves du secondaire (la compétence 3 est supprimée et les deux premières compétences sont reformulées). Cependant, le nouveau programme requiert le développement de deux compétences, dont certaines opérations intellectuelles sont liées à des habiletés essentielles à la pensée historienne, c'est-à-dire les capacités de lire, de critiquer, de contextualiser, de comparer des points de vue ou de comparer des sources historiques variées et parfois contradictoires tout comme le programme précédent l'attendait aussi.

\section{Références}

Altet, M., Desjardins, J., Étienne, R., Paquay, L. et Perrenoud, P. (dir.) (2013). Former des enseignants réflexifs. Obstacles et résistances. Bruxelles : De Boeck.

Apple, M. W. (1992). The text and cultural politics. Educational Researcher, 21(7), 4-19. http://dx.doi.org/10.3102/0013189X021007004

Araujo-Oliveira, A. (2012). Finalités éducatives sous-jacentes aux pratiques des futurs enseignants du primaire : le cas de l'enseignement des sciences humaines et sociales au Québec. Phronesis, 1(4), 84-97. http://dx.doi.org/10.7202/1013239ar

Baquès, M.-C. (2005). Manuels d'histoire et pratiques scolaires en France depuis 1880. Dans J.-L. Jadoulle (dir.), Les manuels scolaires d'bistoire. Passé, présent, avenir (p. 33-53). Louvain-la-Neuve : Université catholique de Louvain.

Bédard, É. et D’Arcy, M. (2011). Enseignement et recherche universitaires au Québec : l'bistoire nationale négligée. Montréal, QC : Coalition pour l'histoire.

Blaser, C. (2007). Fonction épistémique de l'écrit : pratiques et conceptions d'enseignants de sciences et d'histoire du secondaire (Thèse de doctorat, Université Laval, Québec). Repéré à http://hdl.handle.net/20.500.11794/19039

Bouhon, M. (2012). Logiques didactiques et problématisation des contenus dans l'activité de préparation de séquences des enseignants d'histoire. Nouveaux cahiers de la recherche en éducation, 15(1), 69-86. http://dx.doi.org/10.7202/1013380ar 
Bourdieu, P. (1994). Raisons pratiques : sur la théorie de l'action. Paris : Seuil.

Boutonnet, V. (2015a). Pratiques déclarées d'enseignants d'histoire au secondaire en lien avec leurs usages des ressources didactiques et l'exercice de la méthode historique. Revue des sciences de l'éducation de McGill, 50(2-3), 225-246. http://dx.doi.org/10.7202/1036431ar

Boutonnet, V. (2015b). Typologie des usages des ressources didactiques par des enseignants d'histoire au secondaire du Québec. Revue canadienne de l'éducation, 38(1), 1-24. Repéré à http://journals.sfu.ca/cje/index.php/cje-rce/article/view/1926

Braxmeyer, N. (2007). Les pratiques d'enseignement en histoire, géographie et éducation civique au collège. Éducation et formations, (76), 95-104. Repéré à http://www.education.gouv.fr/cid20616/pratiques-d-enseignement-en-histoiregeographie-et-education-civique-au-college.html

Chaubet, P. et Gervais, C. (2014). Analyser l'alternance d'enquêtes entre pratique et théorie pour mieux les provoquer? Quand le concept de pensée réfléchie de Dewey aide à comprendre le développement de la pensée et de l'agir de futurs professionnels. Éducation et francophonie, 42(1), 151-168. http://dx.doi.org/10.7202/1024570ar

Demers, S. (2011). Relations entre le cadre normatif et les dimensions téléologique, épistémologique et praxéologique des pratiques d'enseignants d'bistoire et éducation à la citoyenneté : étude multicas (Thèse de doctorat, Université du Québec à Montréal). Repéré à https://archipel.uqam.ca/5079/

Demers, S., Lefrançois, D. et Éthier, M.-A. (2010). Un aperçu des écrits publiés en français et en anglais depuis 1990 à propos des recherches en didactique sur le développement de la pensée historique au primaire. Dans J.-F. Cardin, M.-A. Éthier et A. Meunier (dir.), Histoire, musées et éducation à la citoyenneté (p. 213-245). Québec, QC : MultiMondes.

Éthier, M.-A., Boutonnet, V., Demers, S. et Lefrançois, D. (2017). Quel sens pour l'bistoire? Analyse critique du nouveau programme d'bistoire du Québec et du Canada. Montréal, QC : M Éditeur.

Gervais, C. et Leroux, M. (2011). Ressources mobilisées par des stagiaires pour enseigner : faible part des savoirs théoriques. Revue des sciences de l'éducation, 37(2), 281-306. http://dx.doi.org/10.7202/1008987ar

Guibert, P. (2013). Socialisations professionnelles et résistances à la professionnalisation chez les enseignants du secondaire. Dans M. Altet, J. Desjardins, R. Étienne, L. Paquay et P. Perrenoud (dir.), Former des enseignants réflexifs. Obstacles et résistances (p. 61-78). Bruxelles : De Boeck.

Haydn, T. (2011). The changing form and use of textbooks in the history classroom in the $21^{\text {st }}$ century: A view from the UK. Dans S. Popp, J. Schumman et M. Schmitz (dir.), Analyzing textbooks: Methodological issues (p. 67-88). Schwalbach : Wochenschau Verlag.

Helgason, P. (2010). Critical thinking and textbook in history: an odd couple? Dans P. Helgason et S. Lässig (dir.), Opening the mind or drawing boundaries? History texts in Nordic schools (p. 163-184). Göttingen : V\&R Unipress.

Jadoulle, J.-L. (2015). Quand des enseignants différents sur le plan de leurs conceptions utilisent un même manuel : quels usages effectifs en classe d'histoire? Didactiques en pratique, 1, 47-57.

Kon, J. H. (1995). Teachers' curriculum decision making in response to a new social studies textbook. Theory and Research in Social Education, 23(2), 121-146. http://dx.doi.org/10.1080/00933104.1995.10505748

Korthagen, F., Loughran, J. et Russell, T. (2006). Developing fundamental principles for teacher education programs and practices. Teaching and Teacher Education, 22(8), 1020-1041. http://dx.doi.org/10.1016/j.tate.2006.04.022

Lautier, N. et Allieu-Mary, N. (2008). La didactique de l'histoire. Revue française de pédagogie, (162), 95-131. Repéré à https://journals.openedition.org/rfp/926

Lavallée, J. (2012). Une histoire javellisée au service du présent. Résultats d’une enquête par sondage réalisée auprès des enseignants d'bistoire du secondaire. Repéré à https://www.coalitionhistoire.org/sites/default/files/une-histoire-javellisee-au-service-du-present.pdf 
Lebrun, J. (2001). Place et rôle du manuel scolaire en sciences humaines dans les planifications d'activités d'enseignementapprentissage des futurs enseignants du primaire à l'Université de Sherbrooke. Dans Y. Lenoir, B. Rey, G.-R. Roy et J. Lebrun (dir.), Le manuel scolaire et l'intervention éducative. Regards critiques sur ses apports et ses limites (p. 161-180). Sherbrooke, QC : Éditions du CRP.

Lee, P. et Shemilt, D. (2003). A scaffold, not a cage: progression and progression models in history. Teaching History, 113, 13-23.

Lenoir, Y. (1991). Des conceptions de l'intervention éducative en sciences humaines dans l'enseignement primaire au Québec et quelques implications. Pédagogies, 4, 43-102.

Lenoir, Y. (2011). Toward a transformation of practices in teacher education. McGill Journal of Education, 46(1), $107-121$. http://dx.doi.org/10.7202/1005672ar

Lévesque, S. (2016). Sondage pancanadien auprès des futurs enseignants d'histoire. Dans M.-A. Éthier et É. Mottet (dir.), Didactiques de l'bistoire, de la géographie et de l'éducation à la citoyenneté. Recherches et pratiques (p. 55-70). Louvain-laNeuve : De Boeck.

Malo, A. (2011). Apprendre en contexte de stage : une dynamique de transformations de répertoire. Revue des sciences de l'éducation, 37(2), 237-255. http://dx.doi.org/10.7202/1008985ar

Martineau, R. (1999). L'histoire à l'école, matière à penser... Paris : L'Harmattan.

Martineau, S., Gervais, C., Portelance, L. et Mukamurera, J. (2008). L'insertion professionnelle des enseignants. Dans L. Portelance, J. Mukamurera, S. Martineau et C. Gervais (dir.), L'insertion dans le milieu scolaire : une phase cruciale du développement professionnel de l'enseignant (p.1-8). Québec, QC : Presses de l'Université Laval.

Ministère de l'Éducation, du Loisir et du Sport. (2007). Programme de formation de l'école québécoise. Enseignement secondaire deuxième cycle. Québec, QC : Gouvernement du Québec.

Ministère de l'Éducation et de l'Enseignement supérieur (MEES). (2017). Programme de formation de l'école québécoise. Enseignement secondaire. Histoire du Québec et du Canada. Troisème et quatrième secondaire. Québec, QC : Gouvernement du Québec.

Moisan, S. (2010). Fondements épistémologiques et représentations sociales d'enseignants d'bistoire du secondaire à l'égard de l'enseignement de l'bistoire et de la formation citoyenne (Thèse de doctorat, Université de Montréal). Repéré à https://papyrus.bib.umontreal.ca/xmlui/handle/1866/4771

Monte-Sano, C. (2011). Learning to open up history for students: Preservice teachers' emerging pedagogical content knowledge. Journal of Teacher Education, 62(3), 260-272. http://dx.doi.org/10.1177/0022487110397842

Morton, T. (2011). Historical thinking in secondary schools: Zones and gardens. Dans P. Clark (dir.), Nerw possibilities for the past: Shaping history education in Canada (p. 195-209). Vancouver, C.-B. : UBC Press.

Niclot, D. et Aroq, C. (2006). Les évolutions récentes des manuels de géographie de l'enseignement secondaire français et les pratiques déclarées des enseignants français. Dans J. Lebrun, J. Bédard, A. Hasni et V. Grenon (dir.), Le matériel didactique et pédagogique : soutien à l'appropriation ou déterminant de l'intervention éducative (p. 55-83). Québec, QC : Presses de l'Université Laval.

Nokes, J. D. (2010). Observing literacy practices in history classrooms. Theory and Research in Social Education, 38(4), 515544. http://dx.doi.org/10.1080/00933104.2010.10473438

Nokes, J. D. (2011). Recognizing and addressing the barriers to adolescents' «reading like historian». The History Teacher, 44(3), 379-404.

Not, L. (1979). Les pédagogies de la connaissance. Toulouse : Privat.

Prost, A. (1996). Douze leçons sur l’histoire. Paris : Seuil.

Reisman, A. (2012). Reading like a historian: A document-based history curriculum intervention in urban high schools. Cognition and Instruction, 30(1), 86-112. http://dx.doi.org/10.1080/07370008.2011.634081 
Reitano, P. et Green, N. C. (2013). Beginning teachers' conceptual understandings of effective history teaching: examining the change from «subject knowers» to «subject teachers». Asia-Pacific Journal of Teacher Education, 41(2), 197-217. http://dx.doi.org/10.1080/1359866x.2013.777027

Repoussi, M. et Tutiaux-Guillon, N. (2012). Les recherches récentes sur les manuels d'histoire : questions méthodologiques et théoriques. Le cartable de Clio, (12), 141-152.

Seixas, P. et Morton, T. (2013). Les six concepts de la pensée historique. Montréal, QC : Groupe Modulo.

Therriault, G. et Harvey, L. (2011). Postures épistémologiques que développent de futurs enseignants de sciences et de sciences humaines lors des cours de formation disciplinaire et pratique : l'apport d'une recherche mixte. Recherches qualitatives, 30(2), 71-95. Repéré à http://www.recherche-qualitative.qc.ca/documents/files/revue/edition reguliere/ numero30(2)/RQ 30(2) Therriault-Harvey.pdf

van Boxtel, C. et van Drie, J. (2017). Engaging students in historical reasoning: The need for dialogic history education. Dans M. Carretero, S. Berger et M. Grever (dir.), Palgrave handbook of research in historical culture and education (p. 573-589). Londres : Palgrave Macmillan. http://dx.doi.org/10.1057/978-1-137-52908-4 30

Vargas, C. (2006). Les manuels scolaires : imperfections nécessaires, imperfections inhérentes et imperfections contingentes. Dans M. Lebrun (dir.), Le manuel scolaire, un outil à multiples facettes (p. 13-35). Québec, QC : Presses de l'Université du Québec.

Vergès, P. (2001). L'analyse des représentations sociales par questionnaires. Revue française de sociologie, 42(3), 537-561. http://dx.doi.org/10.2307/3323032

Vinterek, M. (2010). Different kinds of teaching ressources - Different kinds of learning? Teachers' ends and means. Dans P. Helgason et S. Lässig (dir.), Opening the mind or drawing boundaries? History texts in Nordic schools (p. 121-138). Göttingen : V\&R Unipress.

Wertsch, J. V. (1997). Narrative tools of history and identity. Culture E Psychology, 3(1), 5-20. http://dx.doi.org/10.1177/1354067x9700300101

Wineburg, S. (2001). Historical thinking and other unnatural acts: Charting the future of teaching the past. Philadelphie, PA : Temple University Press.

Wineburg, S., Martin, D. et Monte-Sano, C. (2012). Reading like a historian: Teaching literacy in middle and high School bistory classrooms. New York, NY : Teachers College Press.

Yelle, F. et Déry, C. (2017). L'histoire et la pensée historienne. Dans M.-A. Éthier, V. Boutonnet, S. Demers et D. Lefrançois (dir.), Quel sens pour l'histoire? Analyse critique du nouveau programme d'histoire du Québec et du Canada. (p. 15-47). Montréal, QC : M Éditeur.

Zahorik, J. A. (1991). Teaching style and textbooks. Teaching and Teacher Education, 7(2), 185-196. http://dx.doi.org/10.1016/0742-051x(91)90026-1

\section{Pour citer cet article}

Boutonnet, V. (2018). Usages et fonctions du matériel didactique : pratiques déclarées d'enseignants et de futurs enseignants d'histoire au secondaire. Formation et profession, 26(2), 3-17. http://dx.doi.org/10.18162/fp.2018.462 